\title{
Editorial
}

\section{Theme Articles on Classification and Geometric Data Analysis}

This themed set of articles emerged from presentations and discussions at the International Classification Conference in the University of St Andrews, Scotland, in the summer of 2011. They present inter-esting new directions in methodology.

In their study of the geometry of Canonical Variate Analysis, C.J. Albers and J.C. Gower in "Canonical Analysis: Ranks, Ratios and Fits" pursue generalization of the Mahalanobis distance. They discuss how model fitting and choice of metric are linked. A prime application is to discrimination.

In "Distinguishing and Classifying from n-ary Properties", P. Préa and M. Rolbert study hierarchical classification of n-ary relations. They describe the storage and computational properties of their algorithm.

In "Adaptive Mixture Discriminant Analysis for Supervised Learning with Unobserved Classes", C. Bouveyron develops an adaptive model-based discriminant analysis method using both transductive and inductive learning. A model-based approach is used to estimate the number of classes.

M. Bécue-Bertaut, B. Kostov, A. Morin and G. Naro, in "Rhetorical Strategy in Forensic Speeches: Multidimensional Statistics-Based Methodology" study the internal discursive structure and the develop-ment of the discourse in the case of a prosecutor's concluding speech in a major trial. They use Correspondence Analysis and hierarchical clust-ering for this.

J.C. Gower, N.J. le Roux and S. Lubbe, in "The Canonical Analysis of Distance", generalize Canonical Variate Analysis especially catering for grouped data. The authors introduce the general term Analysis of Distance for this work.

The authors are to be thanked for their work, and appreciation goes to the reviewers for their major contribution as reviewers to this collection of articles. Thanks are due to the Editor-in-Chief of this journal for welcoming this compilation.

Guest editor, Fionn Murtagh

School of Computer Science and Informatics

De Montfort University, UK

fmurtagh@acm.org 